\title{
Comparison of accuracy and stability between disposable arterial blood syringes and pre- heparinized syringe in arterial blood gas analysis: a retrospective study
}

\author{
Xiexiong Zhao \\ Central South University Third Xiangya Hospital \\ Joel Dominic Swai \\ Central South University Third Xiangya Hospital \\ Huiling Chen \\ Central South University Third Xiangya Hospital \\ Huiting Tang \\ Central South University Third Xiangya Hospital \\ Junwen Wang
}

Central South University Third Xiangya Hospital

Jiaying Li

Central South University Third Xiangya Hospital

Nana You

Central South University Third Xiangya Hospital

Yuyan Wu

Central South University Third Xiangya Hospital

Xiaoyu Zhou

Central South University Third Xiangya Hospital

Weihong Jiang ( 2806572418@qq.com )

Central South University Third Xiangya Hospital https://orcid.org/0000-0001-9102-1875

\section{Xiaogang Li}

Central South University Third Xiangya Hospital

\section{Research article}

Keywords: Arterial blood gas analysis; Disposable arterial blood syringe; Pre-heparinized syringe; Retrospective study

Posted Date: September 3rd, 2019 
DOl: https://doi.org/10.21203/rs.2.12304/v1

License: (c) (1) This work is licensed under a Creative Commons Attribution 4.0 International License. Read Full License 


\section{Abstract}

Background Arterial blood gas ( $A B G$ ) analysis is vital in the emergency department. However, the accuracy and stability of some indicators in $A B G$ analysis are debatable compared with venous ones. Disposable arterial blood syringe (DABS) usage is increasing in clinical practice. To compare the accuracy and stability of venous versus arterial blood results and newly emerging DABS versus traditionally used pre-heparinized syringe (PHS) results, we performed this study. Methods This retrospective study was performed at the emergency department of the Third Xiangya Hospital of Central South University. Participants were divided into two groups PHS and DABS, from which venous and arterial blood was drawn and analyzed either in laboratory or ABG-analyzer. Blood sample results were compared by the Pearson correlation coefficient and t-test. By removing extreme results, the sensitivity analysis was conducted. Results A total of 500 patients (64.80\% male) and 400 patients $(65.25 \%$ male) were enrolled in PHS and DABS groups, respectively. Mean differences of blood sample results between venous and arterial from PHS group were: Potassium $=0.318 \pm 0.396 \mathrm{mmol} / \mathrm{L}(P<0.0001)$, Sodium $=2.902 \pm 2.709 \mathrm{mmol} / \mathrm{L}(P<0.0001)$, Chloride $=-7.598 \pm 5.060 \mathrm{mmol} / \mathrm{L}(P<0.0001)$, Hemoglobin $(\mathrm{Hb})=2.212 \pm 7.992 \mathrm{~g} / \mathrm{L}(P<0.0001)$, Hematocrit $(\mathrm{HCT})=1.269 \pm 2.861 \%(P<0.0001)$. Correlation coefficients were: Potassium $=0.855(P<0.0001)$, Sodium $=0.850(P<0.0001)$, Chloride $=0.742(P<0.0001), H b=0.967$ $(P<0.0001)$, and $H C T=0.955(P<0.0001)$. Mean differences from DABS group were:

Potassium $=0.202 \pm 0.276 \mathrm{mmol} / \mathrm{L}(P<0.0001)$, Sodium $=1.187 \pm 2.346 \mathrm{mmol} / \mathrm{L}(P<0.0001)$, Chloride $=-$ $5.336 \pm 6.116 \mathrm{mmol} / \mathrm{L}(P<0.0001), \mathrm{Hb}=-0.898 \pm 9.998 \mathrm{~g} / \mathrm{L}(\mathrm{P}=0.073), \mathrm{HCT}=-0.659 \pm 3.228 \%(P<0.0001)$. Correlation coefficients were: Potassium $=0.923(P<0.0001)$, Sodium $=0.911(P<0.0001)$, Chloride $=0.688$ $(P<0.0001), H b=0.951(P<0.0001)$, and HCT $=0.943$ ( $P<0.0001)$. After sensitivity analysis, the same but more reliable results were observed. Conclusion High accuracy was seen in venous and arterial blood results irrespective of the usage of PHS or DABS for sample collection. DABS showed more accurate and stable results, suggesting its role for future medical use.

\section{Background}

As an important examination for emergency and critical patients, arterial blood gas (ABG) plays an important role in judging patients' oxygen saturation, electrolyte, hemoglobin ( $\mathrm{Hb})$, acid-base balance, and other indicators in a fast manner.

In cases where the patient's condition changes suddenly, ABG analysis can provide clinicians with immediate vital results rather than waiting for the venous blood results to return from the laboratory and delaying the first-aid. ABG analysis is the gold standard method for the assessment of oxygenation and acid-base analysis [1]. In addition, other biochemical indicators, such as blood potassium, sodium, $\mathrm{Hb}$, and hematocrit (HCT), also have important reference values. 
In the past, syringe prepared by $0.4 \%$ heparin sodium solution (pre-heparinized syringe, PHS) was often used to collect $A B G$. Due to various factors, such as heparin saline, the accuracy and stability of blood results in $\mathrm{ABG}$ analysis, such as electrolyte, $\mathrm{Hb}$, and $\mathrm{HCT}$, were often debatable. In clinical work, different doctors still debate on this matter, and in some studies, different scholars also raised different opinions [2-4].

With the development of science and technology, disposable arterial blood syringe (DABS) has been gradually applied in clinical practice. Without the influence of heparin saline, it remains unknown whether the accuracy and stability of ABG analysis will be significantly improved. Therefore, this study has compared the accuracy and stability of indicators collected by different methods (PHS or DABS) for ABG analysis.

\section{Methods}

The whole research process followed the STrengthening the Reporting of OBservational studies in Epidemiology (STROBE) statement. ABG was collected by PHS or DABS (BD Preset ${ }^{T M}, 3 \mathrm{ml}$ ) and analyzed by blood gas analyzer ( $A B L 800$ FLEX), which calibrated automatically in every 1 hour. Venous blood was sent to a central laboratory where blood routine was analyzed by blood routine analyzer (XE-5000), and the electrolyte was analyzed by the automatic biochemical analyzer (Hitachi 7600).

Study population

Patients attending the emergency department of the Third Xiangya Hospital of Central South University between February 01, 2018 and May 30, 2019 were included in the study.

Inclusion criteria区

1. Patients from whom both venous and arterial blood samples were drawn for the analysis of serum electrolyte and blood routine ( $\mathrm{Hb}, \mathrm{HCT})$, which can be gained from the results record;

2. Patients from whom both venous and arterial blood samples were drawn in less than 10 minutes difference [3], which can be identified from the doctor's advice and nursing record.

Exclusion criteria》

1. Patients whose time for venous and arterial blood sample collection was more than ten minutes or not known. 
2. Patients whose method for collecting arterial blood (i.e., PHS or DABS) was unknown, which can be known from the list of expenses of patients recorded in the computer.

\section{Estimation of sample size}

One hundred cases of data were collected in each PHS group and DABS group, and the sample size

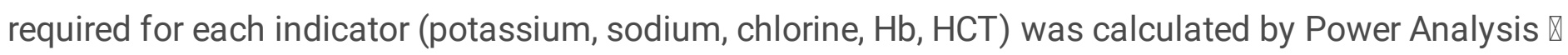
Sample Size (PASS, Version 11.0.7) with parameters $\alpha=0.05$ and $\beta=0.1$ (Yuyan Wu). The target sample size was achieved by expanding the maximum sample size among all indicators by $20 \%$. Finally, 500 cases in the PHS group and 400 cases in DABS group were decided to be collected.

Random sampling method

In the emergency room of our hospital, we began to use DABS instead of PHS for ABG collection since July 2018. To avoid the influence of season and temperature on the ABG analysis results [5], we selected the cases from February to June 2018 for PHS group and February to May 2019 for DABS group, and collected the first 100 cases of every month that met the requirements according to the admission criteria in a consecutive manner [6].

\section{Data collection}

Patients' demographic information, such as patients' ID, gender, age, admission time, admission diagnosis, and blood sample results, such as blood potassium $\left(\mathrm{K}^{+}\right)$, sodium $\left(\mathrm{Na}^{+}\right)$, chlorine $\left(\mathrm{Cl}^{-}\right), \mathrm{Hb}, \mathrm{HCT}$, from artery or vein were collected, as well as the method of collecting ABG. Data were collected by two researchers independently (Huiting Tang, Huiling Chen), and inconsistencies after data checking were confirmed by a third person (Junwen Wang). Abnormal and extreme values found in the data analysis process were further confirmed by a fourth person (Jiaying Li).

\section{Data analysis}

Data were recorded in Microsoft Excel Home edition 2016 spreadsheet and exported to Statistical Package for the Social Sciences version 23.0 (SPSS Inc. Chicago, USA) for analysis. Continuous variables (e.g., blood potassium, sodium, chlorine, $\mathrm{Hb}, \mathrm{HCT}$ ) were presented as mean \pm standard deviation (SD), and categorical variables as percentages (e.g., gender). Box-plot was used to show the mean difference intuitively. Data points more than 1.5 or 3 times the length of the box body from the edge of the box-plot are defined as abnormal values and extreme values, respectively [7]. Pearson's correlation 
coefficient and paired t-test were used to compare the results between venous and arterial. Independent samples t-test was used to compare the results between the PHS group and DABS group. Chi-square test ( $\chi 2)$ was used to test differences between enumeration data. A P-value of less than 0.05 was considered significant. Test efficiency was calculated by Power Analysis $₫$ Sample Size (PASS, Version 11.0.7).

\section{Results}

\section{Basic data characteristics}

According to the requirement of sample size, 500 cases were enrolled in the PHS group, and 400 cases were enrolled in the DABS group. Gender composition ( $64.80 \%$ and $65.25 \%$ of male ratio, respectively, $\mathrm{P}=0.944)$ and age distribution (63.55 \pm 16.82 and $62.86 \pm 15.65$ years, respectively, $\mathrm{P}=0.532)$ in $\mathrm{PHS}$ and DABS group had no statistical difference (Table 1$)$.

Almost all the venous indicators, e.g., $\mathrm{K}^{+}(4.11 \pm 0.72 \mathrm{mmol} / \mathrm{L}$ and $4.09 \pm 0.71 \mathrm{mmol} / \mathrm{L}$, respectively $\mathbb{}$ $\mathrm{P}=0.773), \mathrm{Na}^{+}(138.09 \pm 4.87 \mathrm{mmol} / \mathrm{L}$ and $137.99 \pm 5.13 \mathrm{mmol} / \mathrm{L}$, respectively, $\mathrm{P}=0.749), \mathrm{Hb}(116.14 \pm 31.04$ $\mathrm{g} / \mathrm{L}$ and $118.73 \pm 30.82 \mathrm{~g} / \mathrm{L}$, respectively $\mathbb{P}=0.213)$, and $\mathrm{HCT}(36.39 \pm 9.59 \%$ and $36.18 \pm 9.22 \%$, respectively $\otimes \mathrm{P}=0.742)$, in $\mathrm{PHS}$ and DABS group had no statistical difference except for $\mathrm{Cl}^{-}(101.15 \pm 6.42$ $\mathrm{mmol} / \mathrm{L}$ and $102.28 \pm 6.45 \mathrm{mmol} / \mathrm{L}$, respectively, $\mathrm{P}=0.009$ ) (Table 1).

All the indicators in $A B G$, e.g., $\mathrm{K}^{+}(3.79 \pm 0.75 \mathrm{mmol} / \mathrm{L}$ and $3.89 \pm 0.70 \mathrm{mmol} / \mathrm{L}$, respectively $\mathbb{P}=0.035), \mathrm{Na}^{+}$ (135.19 $\pm 5.02 \mathrm{mmol} / \mathrm{L}$ and $136.80 \pm 5.69 \mathrm{mmol} / \mathrm{L}$, respectively, $\mathrm{P}<0.0001), \mathrm{Cl}^{-}(108.74 \pm 7.42 \mathrm{mmol} / \mathrm{L}$ and

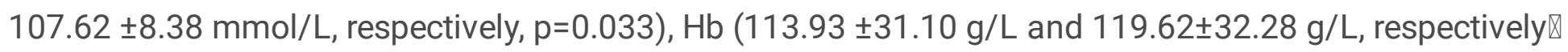
$\mathrm{P}=0.007)$, and HCT $(35.12 \pm 9.36 \%$ and $36.84 \pm 9.72 \%$, respectively $\otimes \mathrm{P}=0.007)$, in $\mathrm{PHS}$ and $\mathrm{DABS}$ group had a statistical difference.

\section{Differences in biochemical indicators between venous results and ABG}

Table 2 shows the difference in blood results between venous blood and arterial blood, which is an important index to reflect the accuracy and stability of $A B G$ analysis. In the PHS group, the difference values were $0.318 \pm 0.396 \mathrm{mmol} / \mathrm{L}(P<0.0001, \beta=0.0001)$ for $\mathrm{K}^{+}, 2.902 \pm 2.709 \mathrm{mmol} / \mathrm{L}(P<0.0001$, $\beta<0.0001)$ for $\mathrm{Na}^{+},-7.598 \pm 5.060 \mathrm{mmol} / \mathrm{L}(P<0.0001, \beta=0.0071)$ for $\mathrm{Cl}^{-}, 2.212 \pm 7.992 \mathrm{~g} / \mathrm{L}(P<0.0001$, $\beta=0.0001)$ for $H b, 1.269 \pm 2.861 \%(P<0.0001, \beta<0.0001)$ for HCT. In the DABS group, the difference values were $0.202 \pm 0.276 \mathrm{mmol} / \mathrm{L}(P<0.0001, \beta<0.0001)$ for $K^{+}, 1.187 \pm 2.346 \mathrm{mmol} / \mathrm{L}(P<0.0001, \beta<0.0001)$ for $\mathrm{Na}^{+},-5.336 \pm 6.116 \mathrm{mmol} / \mathrm{L}(P<0.0001, \beta=0.096)$ for $\mathrm{Cl}^{-},-0.898 \pm 9.998 \mathrm{~g} / \mathrm{L}(P=0.073, \beta=0.021)$ for $\mathrm{Hb},-$ 
$0.659 \pm 3.228 \%(P<0.0001, \beta<0.0001)$ for HCT. The differences between arterial results of PHS and DABS were statistically significant in almost all indicators $\left(\mathrm{K}^{+}, \mathrm{Na}^{+}, \mathrm{Cl}{ }^{-}\right.$, and $\left.\mathrm{HCT}\right)$.

\section{Correlation of biochemical indicators between venous and arterial blood results}

In the group of PHS, the blood results obtained by ABG analysis had a high consistency with those of venous blood at the same time, with Pearson's correlation coefficient of $0.855(P<0.0001)$ for $\mathrm{K}^{+}, 0.850$ $(P<0.0001)$ for $\mathrm{Na}^{+}, 0.742(P<0.0001)$ for $\mathrm{Cl}^{-}, 0.967(\mathrm{P}<0.0001)$ for $\mathrm{Hb}$, and $0.955(\mathrm{P}<0.0001)$ for $\mathrm{HCT}$. Whereas in the group of DABS, the consistency was higher in $\mathrm{K}^{+}$and $\mathrm{Na}^{+}$, with correlation coefficient of $0.923(P<0.0001)$ and $0.911(P<0.0001)$, respectively, except for $\mathrm{Cl}^{-}(0.688, \mathrm{P}<0.0001), \mathrm{Hb}(0.951$, $\mathrm{P}<0.0001)$, and HCT $(0.943, \mathrm{P}<0.0001)$ (Table 3).

\section{Sensitivity analysis}

Through the analysis of box-plot, some abnormal values and extreme values were found, and some extreme values were quite different. However, to minimize the possibility of data entry errors, data were rechecked by multiple people. The possible reasons were measurement errors, special conditions of patients, or others. These abnormal and extreme values may have a great impact on the results. So, a sensitivity analysis was conducted by removing all the extreme values.

By comparison, there were 17 extreme values in the PHS group and 31 extreme values in the DABS group. After removing them, the average difference of the relative indicators between venous results and $A B G$ collected by PHS or DABS was almost unaffected, but the standard deviation was obviously reduced. The results are shown in Table 4. In the PHS group, the adjusted difference values were $0.334 \pm 0.284 \mathrm{mmol} / \mathrm{L}$ $(P<0.0001)$ for $\mathrm{K}^{+}, 2.899 \pm 2.646 \mathrm{mmol} / \mathrm{L}(\mathrm{P}<0.0001)$ for $\mathrm{Na}^{+},-7.359 \pm 4.542 \mathrm{mmol} / \mathrm{L}(\mathrm{P}<0.0001)$ for $\mathrm{Cl}^{-}$, $2.211 \pm 6.018 \mathrm{~g} / \mathrm{L}(\mathrm{P}<0.0001)$ for $\mathrm{Hb}$, and $1.250 \pm 2.371 \%(P<0.0001)$ for HCT. In the $D A B S$ group, the adjusted difference values were $0.193 \pm 0.255 \mathrm{mmol} / \mathrm{L}(\mathrm{P}<0.0001)$ for $\mathrm{K}^{+}, 1.158 \pm 2.219 \mathrm{mmol} / \mathrm{L}$ $(P<0.0001)$ for $\mathrm{Na}^{+},-5.620 \pm 4.213 \mathrm{mmol} / \mathrm{L}(P<0.0001)$ for $\mathrm{Cl}^{-},-0.404 \pm 5.242 \mathrm{~g} / \mathrm{L}(P=0.140)$ for $\mathrm{Hb}$, and $0.434 \pm 1.953 \%(P<0.0001)$ for HCT.

At the same time, the adjusted correlation of results between venous and arterial blood samples also increased. The correlation coefficients were $\mathrm{K}^{+}(0.916, \mathrm{P}<0.0001), \mathrm{Na}^{+}(0.852, \mathrm{P}<0.0001), \mathrm{Cl}^{-}(0.793$, $\mathrm{P}<0.0001), \mathrm{Hb}(0.981, \mathrm{P}<0.0001), \mathrm{HCT}(0.969, \mathrm{P}<0.0001)$ in the PHS group and $\mathrm{K}^{+}(0.933, \mathrm{P}<0.0001), \mathrm{Na}^{+}$ 
(0.921, $\mathrm{P}<0.0001), \mathrm{Cl}^{-}(0.819, \mathrm{P}<0.0001), \mathrm{Hb}(0.986, \mathrm{P}<0.0001), \mathrm{HCT}(0.978, \mathrm{P}<0.0001)$ in the DABS group (Table 5).

\section{Discussion}

$A B G$ analysis is a very important examination in acute and critical cases. In the past, PHS was used to collect ABG. However, its consistency with venous blood was often questioned by the clinicians [8]. Our study explored the accuracy and stability of related indicators of venous blood and ABG collected by PHS and DABS in retrospective research and found that irrespective of the method used for collecting $A B G$ blood sample, the consistency was high, especially ABG collected by DABS.

From the basic characteristics, there was almost no statistical difference in age, gender, and venous blood results between PHS group and DABS group, suggesting that the population composition of the two groups was comparable though the collection duration was different. Because of the different ways of collecting arterial blood, the difference in arterial biochemical indicators between the two groups is what our study explored.

The qualitative analysis revealed that the correlation coefficient of the blood results between ABG and venous blood was markedly high, no matter which method, PHS or DABS, was used to collect ABG. It is worth mentioning that DABS had a significantly higher consistency in $\mathrm{K}^{+}$and $\mathrm{Na}^{+}$, while PHS seemed to have a higher consistency in $\mathrm{Cl}^{-}, \mathrm{Hb}$, and $\mathrm{HCT}$. But after the sensitivity analysis, all five blood results had higher correlation coefficients in DABS after removing the extreme values.

The quantitative analysis, that is, from the difference of related indicators between venous blood and arterial blood, showed that all the results of arterial blood collected with DABS had less mean differences compared with the venous blood, especially in $\mathrm{Hb}$, whose mean difference was too small to have a statistical difference, suggesting that DABS had a higher accuracy in collecting ABG. Regarding standard deviation, DABS had a smaller standard deviation in $\mathrm{K}^{+}$and $\mathrm{Na}^{+}$. While PHS appeared to have a smaller standard deviation in $\mathrm{Cl}^{-}, \mathrm{Hb}$, and $\mathrm{HCT}$. But after the sensitivity analysis, all five blood results had a smaller standard deviation in DABS after removing the extreme values, suggesting that DABS had higher stability.

The possible reasons for the difference in arteriovenous blood samples are as follows: Firstly, the use of heparin sodium saline dilutes the arterial blood samples in different degrees at each time of ABG 
collection. Therefore, the blood results of the arterial blood samples taken with PHS are generally lower than those of the venous blood samples. At the same time, the amount of heparin saline attached to the syringe tube wall is uncertain each time. This results in fluctuations with true value, which is consistent with the conclusion of this study. As a result, using DABS to collect ABG avoids such an error, so DABS is more accurate and stable. Secondly, there may be inherent differences in the biochemical indicators of arteriovenous blood [9], such as blood potassium. After capillary microcirculation, metabolites, such as lactic acid, entered the vein, making the $\mathrm{PH}$ of venous blood more acidic, affecting $\mathrm{H}^{+}-\mathrm{K}^{+}$exchange, and resulting in higher potassium in venous blood than that in arterial blood. Further experiments are needed to analyze the arteriovenous blood on the same instrument at the same time. Thirdly, there could be systematic errors in the arteriovenous blood detection instrument, which might be the reason why our findings are some kind of different from other researches $[2,3]$ where sodium and potassium showed no significant difference between venous and arterial but $\mathrm{Hb}$ did. Fourthly, hemolysis may occur during blood collection [10]. Fifthly, potassium will release from platelets during coagulation [11]. Lastly, heparin sodium itself can bind positive ions. Heparin molecule contains sulfate groups and carboxyl groups, which is a strong acidic polyanion, and is capable of reacting with a cationic salt, including $\mathrm{Ca}^{2+}, \mathrm{Na}^{+}, \mathrm{K}^{+}$, etc. [12], thus reducing the corresponding cationic concentration.

It is strange that there are more extreme values in DABS group than in the PHS group. But it is not difficult to understand that the DABS group's data is more stable, so the quartile spacing is smaller, and the data falling three times out the quartile spacing naturally increases.

It is worth noting that when sensitivity analysis was conducted by removing the extreme values, $\mathrm{Hb}$ and HCT changed greatly, even reversing the previous conclusions. It attracted our attention on how extreme values affect $\mathrm{Hb}$ and $\mathrm{HCT}$. Therefore, we further analyzed the extreme values of $\mathrm{Hb}$ and $\mathrm{HCT}$. We found that the extreme values of $\mathrm{Hb}$ and HCT were highly consistent in both PHS and DABS groups (Figure 1a, $1 b, 1 d, 1 e)$. For the extreme values, whether in PHS or DABS group, annihilation and gastrointestinal hemorrhage were the main common characteristics of these abnormal values. Chest pain, uremia, and others were also the common characteristics of the abnormal values (Figure 1c, 1f). Among them, the differences in $\mathrm{Hb}$ between venous blood and arterial in patients with gastrointestinal hemorrhage was obvious, which had never been reported before. We speculate that in the course of acute hemorrhage, the microcirculation of the body compensates for the replenishment of fluid in the blood vessels to dilute the venous blood, especially, the red blood cells could not pass through the blood vessel wall. As a result, $\mathrm{Hb}$ and HCT in the arteriovenous blood are quite different even though they are sampled at the same time. We call for a more in-depth study of the causes and mechanisms of extreme values in blood gas. It also suggests that when patients suffered from annihilation, gastrointestinal hemorrhage, chest pain, uremia, the indicators in $A B G$ might have obviously deviated from the true value. 


\section{Limitation}

In the study, values tested from venous blood were regarded as the golden standard, but there may be measurement errors in venous blood itself, which inevitably leads to bias. This is a real-world retrospective analysis, in which ABG and venous values are common emergency indicators, so it could not achieve absolute control like arterial-arterial comparison or venous-venous comparison. Some studies pointed out that venous blood gas could be used to replace ABG [13], but it could not judge the oxygen and carbon dioxide indicators. Although those studies have given the relevant formula $[14,15]$, the calculation is relatively complicated, and the results were not visualized. So, the accuracy and stability between $A B G$ and venous blood indicators need to be further confirmed by prospective multi-center research.

\section{Conclusions}

There is a high correlation of results in venous and ABG blood samples collected in PHS or DABS groups, although the difference is statistically significant. In some indicators (such as $\mathrm{Hb}, \mathrm{HCT}$ ), these differences may be ignored and regarded as of no clinical significance. It might be easy to estimate intravenous blood results only by the average values of $A B G$ results. However, what confuses clinicians is the deviation of values. The reason for such deviations is still debatable. However, DABS is more accurate and stable than PHS and may become a consensus method in judging the patients' condition of acute diseases in the future. We still need to pay attention to special conditions, such as gastrointestinal bleeding, annihilation, chest pain, uremia, etc., that may have an extreme impact on ABG outcomes.

\section{List Of Abbreviations}

$A B G$, arterial blood gas; PHS, pre-heparinized syringe; DABS, disposable arterial blood syringe; SD, standard deviation; $\mathrm{Cl}$, confidence interval

\section{Declarations}

\section{Ethics approval and consent to participate}

Not applicable.

\section{Consent for publication}

Not applicable.

\section{Availability of data and material}


The datasets used and analysed during the current study are available from the corresponding author on reasonable request.

\section{Competing interests}

The authors declare that they have no competing interests

\section{Funding}

The study was supported by the National Natural Science Foundation of China (NSFC) Projects (NO. 81670335, and 81800271), the New XiangYa Talent Projects of the Third XiangYa Hospital of Central South University (NO.20170304) and the Natural Science Foundation of Hunan Province (N0.2019JJ50920).

\section{Authors' contributions}

XXZ for the research design and writing the article, JDS for data analysis and writing the article, HLC and HTT for data collection, JWW and JYL for data checking, YYW for sample size estimating, NNY and XYZ for data analysis, XGL and WHJ for quality control and final approval of the version to be published. All authors read and approved the final manuscript.

\section{Acknowledgements}

Not applicable.

\section{References}

1. Zeserson E, Goodgame B, Hess JD, Schultz K, Hoon C, Lamb K, Maheshwari V, Johnson S, Papas M, Reed J et al: Correlation of Venous Blood Gas and Pulse Oximetry With Arterial Blood Gas in the Undifferentiated Critically III Patient. Journal of intensive care medicine 2018, 33(3):176-181. 2. Quinn LM, Hamnett N, Wilkin R, Sheikh A: Arterial blood gas analysers: accuracy in determining haemoglobin, glucose and electrolyte concentrations in critically ill adult patients. $\mathrm{Br} J$ Biomed Sci 2013, 70(3):97-100. 3. Johnston H, Murphy R: Agreement between an arterial blood gas analyser and a venous blood analyser in the measurement of potassium in patients in cardiac arrest. Emergency Medicine Journal : EMJ 2005, 22(4):269-271. 4. Zhang JB, Lin J, Zhao XD: Analysis of bias in measurements of potassium, sodium and hemoglobin by an emergency department-based blood gas analyzer relative to hospital laboratory autoanalyzer results. PLoS ONE 2015, 10(4):e0122383. 5. Hussein H, Aamer A: Influence of different storage times and temperatures on blood gas and acid-base balance in ovine venous blood. Open Veterinary Journal 2013, 3(1):1-7. 6. Hao Y, Liu J, Smith SC, Huo Y, Fonarow GC, Ge J, Liu J, Taubert KA, Morgan L, Guo Y et al: Rationale and design of the improving Care for Cardiovascular Disease in China (CCC) project: a national registry to improve management of atrial fibrillation. BMJ Open 2018, 8(7):e020968. 7. Zhang W: SPSS Basic Course of Statistical Analysis, 3 edn. China: Higher Education Press; 2017. 8. Budak YU, Huysal K, Polat M: Use of a blood gas analyzer and a laboratory autoanalyzer 
in routine practice to measure electrolytes in intensive care unit patients. BMC Anesthesiology 2012, 12:17. 9. Awasthi S, Rani R, Malviya D: Peripheral venous blood gas analysis: An alternative to arterial blood gas analysis for initial assessment and resuscitation in emergency and intensive care unit patients. Anesthesia, Essays and Researches 2013, 7(3):355-358. 10. Mrazek C, Simundic AM, Wiedemann H, Krahmer F, Felder TK, Kipman U, Hoppe U, Haschke-Becher E, Cadamuro J: The relationship between vacuum and hemolysis during catheter blood collection: a retrospective analysis of six large cohorts. Clin Chem Lab Med 2017, 55(8):1129-1134. 11. Hartmann RC, Auditore JV, Jackson DP: Studies on thrombocytosis. I. Hyperkalemia due to release of potassium from platelets during coagulation. Journal of Clinical Investigation 1958, 37(5):699-707. 12. Chemical Book: Heparin sodium. https://www.chemicalbook.com/ChemicalProductProperty_EN_CB0439583.htm. Accessed 16 July 2019. 13. Shirani F, Salehi R, Naini AE, Azizkhani R, Gholamrezaei A: The effects of hypotension on differences between the results of simultaneous venous and arterial blood gas analysis. Journal of Research in Medical Sciences : The Official Journal of Isfahan University of Medical Sciences 2011, 16(2):188-194. 14. Kim BR, Park SJ, Shin HS, Jung YS, Rim H: Correlation between peripheral venous and arterial blood gas measurements in patients admitted to the intensive care unit: A single-center study. Kidney Research and Clinical Practice 2013, 32(1):32-38. 15. Treger R, Pirouz S, Kamangar N, Corry D: Agreement between Central Venous and Arterial Blood Gas Measurements in the Intensive Care Unit. Clinical Journal of the American Society of Nephrology : CJASN 2010, 5(3):390-394.

\section{Table And Figure Legends}

Table 1. Characteristics of basic indicators by two methods for ABG.

Table 2. Differences of biochemical indicators between venous results and ABG by two methods.

Table 3. Correlation of biochemical indicators between venous results and ABG by two methods.

Table 4. Adjusted differences of biochemical indicators between venous results and ABG by two methods after removing extreme value.

Table 5. Adjusted correlation of biochemical indicators between venous results and ABG by two methods after removing extreme value.

Figure 1. Analysis of extreme values in $\mathrm{Hb}$ and $\mathrm{HCT}$ from $\mathrm{ABG}$ by two methods. $\mathrm{a}, \mathrm{b}$ show box-plots of $\mathrm{Hb}$ and HCT difference between venous results and ABG by PHS, respectively; c shows cause analysis of extreme values in $\mathrm{Hb}$ and $\mathrm{HCT}$ from $\mathrm{a}$ and $\mathrm{b}$; $\mathrm{c}$, d show box-plots of $\mathrm{Hb}$ and $\mathrm{HCT}$ difference between venous results and $A B G$ by DABS, respectively; $f$ shows cause analysis of extreme values in $\mathrm{Hb}$ and $\mathrm{HCT}$ from $c$ and d. o for abnormal values and * for extreme values in box-plots. Hb, hemoglobin; HCT, hematocrit; $A B G$, arterial blood gas; PHS, pre-heparinized syringe; DABS, disposable arterial blood syringe.

\section{Tables}


Due to technical limitations, tables 1-5 are only available as a download in the supplemental files section.

\section{Figures}

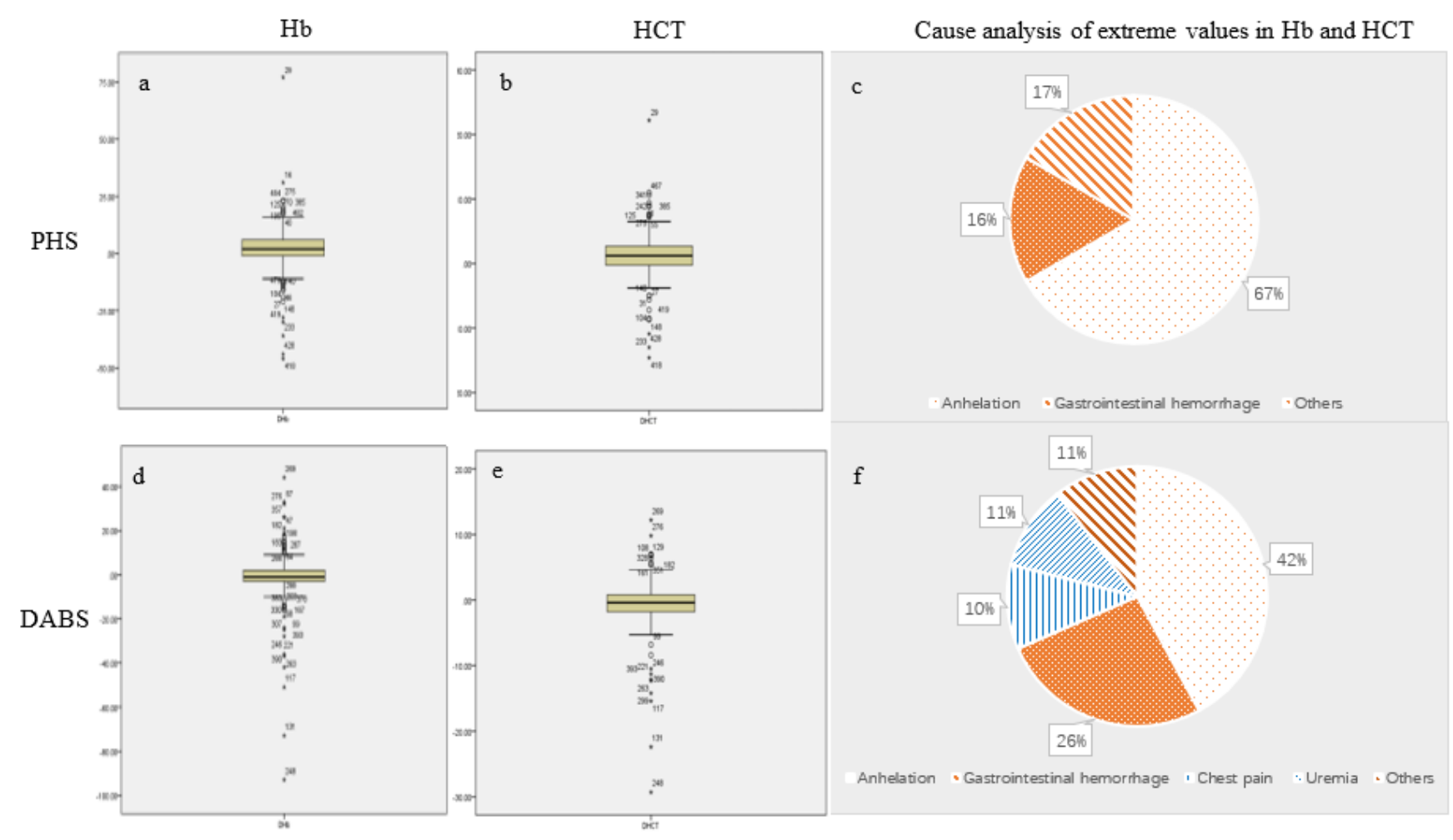

\section{Figure 1}

Analysis of extreme values in $\mathrm{Hb}$ and $\mathrm{HCT}$ from $\mathrm{ABG}$ by two methods.

\section{Supplementary Files}

This is a list of supplementary files associated with this preprint. Click to download.

- supplement1.pdf 